\title{
Methylation Profiles of Multiple CpG Island Loci in Extrahepatic Cholangiocarcinoma Versus Those of Intrahepatic Cholangiocarcinomas
}

\author{
Baek-Hee Kim, MD; Nam-Yun Cho, MSc; Minhee Choi, BSc; Sun Lee, MD; Ja June Jang, MD; Gyeong Hoon Kang, MD
}

- Context.-CpG island hypermethylation is attracting attention because of its importance as a tumor marker and its potential mechanism for the development of human cancers. Extrahepatic cholangiocarcinoma has been poorly investigated with respect to $\mathrm{CpG}$ island hypermethylation, and the number of genes known to be methylated in extrahepatic cholangiocarcinomas is fewer than 20.

Objective.-To generate methylation profiles of $24 \mathrm{CpG}$ island loci in extrahepatic cholangiocarcinomas, to correlate methylation findings with clinicopathologic findings, and to compare these findings with those of intrahepatic cholangiocarcinomas.

Design.-Sixty-three extrahepatic cholangiocarcinomas and 48 intrahepatic cholangiocarcinomas were investigated for hypermethylation in $24 \mathrm{CpG}$ island loci by using methylation-specific polymerase chain reaction.

Results.-A total of $61(96.8 \%)$ of 63 extrahepatic cholangiocarcinomas showed hypermethylation in at least one of the examined loci, and a high methylation frequency

A denocarcinoma of the extrahepatic bile duct (extrahepatic cholangiocarcinoma [ECC]) is relatively rare and contributes less than $0.2 \%$ to the incidence of invasive cancers in the United States. ${ }^{1}$ The condition causes obstruction of the extrahepatic bile duct, which results in jaundice, cola-colored urine, and pruritus and spreads by early direct extension to adjacent tissues and organs. ${ }^{2}$ Distant metastases have been reported in $30 \%$ to $70 \%$ of cases, and patients with ECC have a poor prognosis (2-year survival rate, $23 \%) .{ }^{3}$ Little is known about the causes of ECC other than its frequent association with ulcerative colitis, primary sclerosing cholangitis, and choledochal cysts. So far, the molecular events of ECC carcinogenesis are not well understood. Known genetic changes in ECC include mutations of $p 53, p 16$, and KRAS; amplifications of $c E R B 2$, EGFR, and CMET; and loss of DPC4. ${ }^{4-7}$

Accepted for publication November 29, 2006.

From the Department of Pathology (Drs Kim, Jang, and Kang) and Laboratory of Epigenetics, Cancer Research Institute (Mr Cho and Ms Choi), Seoul National University College of Medicine, and Department of Pathology, Kyung Hee University College of Medicine (Dr Lee), Seoul, South Korea.

The authors have no relevant financial interest in the products or companies described in this article.

Reprints: Gyeong Hoon Kang, MD, Department of Pathology, Seoul National University College of Medicine, 28 Yongon-dong, Chongnogu, Seoul 110-744 South Korea (e-mail: ghkang@snu.ac.kr). was seen in HOXA1 $(95.2 \%)$, HPP1 $(69.8 \%)$, and NEUROG1 (61.9\%). The number of methylated CpG island loci was greater in extrahepatic cholangiocarcinomas with nodal metastasis than in those without nodal metastasis $(P=$ $.047)$, and hypermethylation of TIG1 was closely associated with nodal metastasis of extrahepatic cholangiocarcinomas $(P=.007) . C D H 1$ and NEUROG1 were more frequently methylated in extrahepatic cholangiocarcinoma than in intrahepatic cholangiocarcinoma, whereas CHFR, GSTP1, IGF2, MGMT, MINT31, p14, and RBP1 were more frequently methylated in intrahepatic cholangiocarcinoma: the differences was statistically significant $(P<.05)$.

Conclusions. - A close relationship exists between CpG island hypermethylation and nodal metastasis of extrahepatic cholangiocarcinomas. Methylation profiles of extrahepatic cholangiocarcinomas are somewhat similar to but distinct from those of intrahepatic cholangiocarcinomas.

(Arch Pathol Lab Med. 2007;131:923-930)

Promoter CpG island hypermethylation is closely associated with gene inactivation and is a common finding in human cancers, regardless of tissue type. However, tissue type variations of $\mathrm{CpG}$ island hypermethylation are known to occur with regard to the methylated gene type, the methylation frequencies of specific genes, and the overall methylation extent. ${ }^{8,9}$ Moreover, it is believed that different human cancer types have unique profiles of $\mathrm{CpG}$ island hypermethylation, and that although some gene changes are shared, others are specific to the type of cancer. Even different histologic subtypes of cancers within a given organ appear to have different methylation profiles. ${ }^{10}$ Recent studies have documented CpG island hypermethylation in some genes in ECC, but to date fewer than 20 genes have been reported to be hypermethylated in ECC. ${ }^{11-18}$ Because about $40 \%$ of human genes have CpG islands on their promoter sequences, it is believed that many unknown genes are methylated in ECC.

In addition to its potential role in gene inactivation, $\mathrm{CpG}$ island hypermethylation is now gaining attention as a molecular marker for tumor detection and for the prediction of cancer development or progression. ${ }^{19,20}$ Extrahepatic cholangiocarcinoma is difficult to visualize on ultrasonography or on a standard computed tomographic scan; hilar cholangiocarcinoma is a diagnostic challenge and is difficult to differentiate from sclerosing cholangitis.

Methylation Profiles of Bile Duct Adenocarcinoma-Kim et al 923 


\begin{tabular}{|c|c|}
\hline \multicolumn{2}{|c|}{$\begin{array}{l}\text { Table 1. Clinicopathologic Characteristics of } \\
\text { Extrahepatic Cholangiocarcinomas* }\end{array}$} \\
\hline Characteristic & Data \\
\hline Cases, No. & 63 \\
\hline Men/women, No. & $43 / 20$ \\
\hline Age, mean (median, range), y & $62.9(64,29-81)$ \\
\hline \multicolumn{2}{|l|}{ Location, No. (\%) } \\
\hline Proximal & $33(52.4)$ \\
\hline Distal & $30(47.6)$ \\
\hline \multicolumn{2}{|l|}{ Gross type, No. $(\%)^{*}$} \\
\hline Papillary & $12(19)$ \\
\hline Nodular & $24(38.1)$ \\
\hline Scirrhous & $12(19)$ \\
\hline Diffuse & $15(23.8)$ \\
\hline \multicolumn{2}{|l|}{ Differentiation, No. (\%) } \\
\hline Papillary & $12(19)$ \\
\hline Tubular, well & $2(3.2)$ \\
\hline Tubular, moderate & $39(61.9)$ \\
\hline Tubular, poor & $10(15.9)$ \\
\hline \multicolumn{2}{|c|}{ Tumor, node, metastasis stage, No. $(\%)$} \\
\hline \multicolumn{2}{|l|}{ Pathologic } \\
\hline IA & $5(7.9)$ \\
\hline $\mathrm{IB}$ & $20(31.7)$ \\
\hline$\| A$ & $17(26.9)$ \\
\hline IIB & $14(22.2)$ \\
\hline III & $6(9.5)$ \\
\hline IV & $1(1.5)$ \\
\hline \multicolumn{2}{|l|}{ Clinical } \\
\hline IA & $19(30.1)$ \\
\hline IB & $27(42.8)$ \\
\hline IIA & $14(22.2)$ \\
\hline IIB & $12(19.0)$ \\
\hline III & $1(1.5)$ \\
\hline IV & 0 \\
\hline
\end{tabular}

* Classified according to World Health Organization Classification of Tumours. ${ }^{25}$

Moreover, tissue-based diagnoses are difficult, except in advanced cases, ${ }^{21}$ and cytologic examinations have low sensitivity for the detection of ECC 22,23 ; this low sensitivity might lead to delays in the administration of proper treatment. Thus, DNA methylation markers that specifically distinguish ECC from noncancerous biliary epithelia would be of great value for the detection of malignancy when a limited volume of tissue or limited cell numbers are available. Therefore, DNA methylation markers may provide a powerful means of detecting ECC based on genetic material obtained from tumors directly or from bile juice or serum.

The purpose of this study was to investigate the prevalence of hypermethylation in 63 cases of ECC by using a panel of $24 \mathrm{CpG}$ island loci, which have been previously demonstrated to be hypermethylated in other human cancer tissue types. We sought to identify new methylation markers for ECC detection. In addition, we compared the methylation profiles of ECCs with those of intrahepatic cholangiocarcinomas (ICCs).

\section{MATERIALS AND METHODS}

Formalin-fixed, paraffin-embedded tissue samples were obtained from 63 patients who had undergone curative surgical resection for ECC at Seoul National University Hospital between January 2001 and December 2003. Pathologic stages were determined by using the current tumor, node, metastasis classification, ${ }^{24}$ and tumor grades were determined by using the World Health Organization classification system for ECC. ${ }^{25}$ Extrahepatic cholangiocarcinomas were subclassified into proximal ECCs if they arose from the suprapancreatic portion of the bile duct and as distal ECCS if they arose from the intrapancreatic or retroduodenal bile duct regions. Table 1 summarizes the clinicopathologic characteristics of the 63 ECC cases. In addition, 48 previously studied ICC tumor samples ${ }^{26}$ were also reexamined during this study. A total of 38 cases of benign biliary epithelia were included as controls. These normal bile duct epithelia were obtained from the patients with nonneoplastic liver or biliary diseases, including hepatolithiasis $(n=24)$, Caroli disease $(n=6)$, and liver cirrhosis $(\mathrm{n}=8)$. Through microscopic examination, we confirmed that the selected archival tissue blocks of the control group did not have dysplastic foci in the biliary epithelia.

This study was approved by the institutional review board.

\section{DNA Extraction}

Tumor portions showing a ratio of tumor cells to nontumor cells greater than 30:70 were selected after examination of histologic slides under a microscope. Areas of interest were delineated on paraffin-tissue blocks by using a marker pen and were dissected from tissue blocks by using a scalpel into microtubes.

\begin{tabular}{|cll|}
\hline Table 2. Primer Sequences and Polymerase Chain Reaction Conditions for Methylation-Specific Polymerase Chain \\
Reaction Analysis*
\end{tabular}

* $\mathrm{m}$ indicates methylated; $\mathrm{u}$, unmethylated. 
The scraped tissues were dewaxed with xylene and alcohol and lysed in lysis buffer solution containing proteinase $\mathrm{K}$.

\section{Bisulfite Modification and Methylation-Specific Polymerase Chain Reaction}

Sodium bisulfite conversion of genomic DNA was performed as follows. Briefly, $18 \mu \mathrm{L}$ of genomic DNA (5 $\mu \mathrm{g})$ was denatured at $100^{\circ} \mathrm{C}$ for 10 minutes and then incubated in $0.3 \mathrm{M} \mathrm{NaOH}$ at $42^{\circ} \mathrm{C}$ for 20 minutes. Bisulfite conversion was performed by using $2.5 \mathrm{M}$ sodium metabisulfite solution at $50^{\circ} \mathrm{C}$ for 16 hours in the dark. DNA was purified from bisulfite solutions by using QIAamp Viral RNA Mini Kits (Qiagen, Valencia, Calif) according to the manufacturer's recommendations, except that each sample was loaded twice onto each spin column. After loading the entire samples, spin columns were washed, and samples were eluted twice by using $40 \mu \mathrm{L}$ of elution buffer. Eluted samples were then desulfonated by incubating them in $0.077 \mathrm{M} \mathrm{NaOH}$ solution at room temperature for 15 minutes. After neutralization with $1 \mathrm{M}$ $\mathrm{HCl}$, DNA was purified a second time by using QIAamp Viral RNA Mini Kits (Qiagen). The eluted DNA samples so obtained were used for methylation-specific polymerase chain reaction (MSP).

Methylation-specific PCR was used to examine the methylation statuses of $24 \mathrm{CpG}$ island loci $(A P C, B C L 2, C A C N A 1 G, C D H 1$, CHFR, COX2, GSTP1, HOXA1, HPP1, IGF2, MGMT, MINT1, MINT2, MINT31, NEUROG1, p14, p16, RARB2, RASSF1A, RBP1, RUNX3, THBS1, TIG1, and TIMP3). We designed oligonucleotide primers for 9 CpG island loci (BCL2, CACNA1G, CHFR, HOXA1, HPP1, IGF2, NEUROG1, RBP1, and TIG1); the primer sequences of each $\mathrm{CpG}$ island are provided in Table 2 . The primer sequences of the other $\mathrm{CpG}$ island loci have been reported elsewhere. ${ }^{26-28}$ Methylation-specific PCR was performed in $30-\mu \mathrm{L}$ reaction volumes containing $1 \times \mathrm{PCR}$ buffer $\left(16.6 \mathrm{mM}(\mathrm{NH} 4)_{2} \mathrm{SO}_{4} ; 67 \mathrm{mM}\right.$ Tris, $\mathrm{pH} 8.8 ; 6.7 \mathrm{mM} \mathrm{MgCl}_{2} ; 10 \mathrm{mM} \beta$-mercaptoethanol), deoxyribonucleotide phosphate (each at $1 \mathrm{mM})$, primers (10 pmol each), and bisulfite-modified DNA (30-50 ng). Reactions were hot-started at $97^{\circ} \mathrm{C}$ for 5 minutes before addition of $1 \mathrm{U}$ of Taq polymerase (Takara Shuzo, Kyoto, Japan). Amplifications were carried out in a Thermal cycler (Perkin-Elmer, Foster City, Calif) for 35 cycles, followed by a final 10-minute extension. The annealing temperatures of the different primers are given in Table 2. The PCR products obtained were electrophoresed in $2.5 \%$ agarose gels and visualized under ultraviolet illumination after staining with ethidium bromide. Samples showing signals of more than that shown by $3.5 \mathrm{ng}$ of 100-base pair size marker were scored as methylated. Results were scored by 2 independent observers, and samples showing weak or faint signals were subjected to repeated MSP assays. For each MSP reaction, we used normal lympho- cyte DNA treated with Sss1 methyl transferase as a positive control and distilled water without template DNA as a negative control.

\section{Sequencing Analysis}

The PCR products were purified by using the JETSORB gel extraction kit (Genomed, Bad Oeynhausen, Germany) and cloned into the pGEM-T easy vector (Promega, Madison, Wis). Five colonies were selected from each sample and expanded in liquid culture. Plasmid DNA was purified from individual clones by using AccuPrep plasmid extraction kit (Bioneer, Seoul, South Korea). The inserted PCR fragments were sequenced with both M13R3 primer using an Applied Biosystems automated sequencer.

\section{Statistical Analysis}

The Fisher exact test was used to compare the hypermethylation frequencies of $\mathrm{CpG}$ island loci in ECC and ICC or normal bile duct tissues. To compare means between groups, analysis of variance test or a Student $t$ test was used. Actuarial survival rates were evaluated by using the Kaplan-Meier method, and differences were tested by using the log-rank test. SPSS software was used for all analyses (SPSS for Windows Release, version 11.0, SPSS, Chicago, Ill). $P$ values less than .05 indicated statistical significance.

\section{RESULTS}

\section{Methylation Frequencies of CpG Island Loci in ECC Versus Those in ICC or Normal Bile Duct Tissues}

Sixty-three cases of ECC and 48 cases of ICC were examined for the methylation status of $24 \mathrm{CpG}$ island loci. To rule out false amplification of $9 \mathrm{CpG}$ island loci that we designed oligonucleotide primers for, we sequenced the representative MSP products of each CpG island locus and confirmed that MSP reactions amplified the targeted genomic fragments and $\mathrm{CpG}$ sites located inside the amplified genomic fragments showed extensive methylation. Figure 1 shows a representative example of MSP analysis, and Figure 2 shows the detailed results of the methylation statuses of $24 \mathrm{CpG}$ island loci in ECC, ICC, and normal bile duct tissues. Methylation frequencies at each $\mathrm{CpG}$ island locus varied from $0 \%$ to $95.2 \%$ in ECC and from $0 \%$ to $83.3 \%$ in ICC (Table 2). A total of $61(96.8 \%$ ) of the 63 ECCs and a total of $47(97.9 \%)$ of the 48 ICCs showed methylation in at least 1 of $24 \mathrm{CpG}$ island loci (Table 3).

\begin{tabular}{|c|c|c|}
\hline Annealing Temperature, ${ }^{\circ} \mathrm{C}$ & Nucleotide Position & Accession No. \\
\hline 59 & $32340-32236$ & AC009267 \\
\hline 55 & $32342-32230$ & \\
\hline 59 & $27821-27924$ & AC004590 \\
\hline 59 & $27777-27876$ & \\
\hline 55 & $61962-62070$ & AC127070 \\
\hline 59 & $62055-62176$ & \\
\hline 59 & 78608-78706 & AC004079 \\
\hline 55 & 78664-78778 & \\
\hline 61 & $181-296$ & AF264150 \\
\hline 59 & $195-296$ & \\
\hline 59 & $108634-108727$ & AF132217 \\
\hline 59 & 108613-108733 & \\
\hline 59 & $75366-75483$ & AC005738 \\
\hline 59 & $75427-75545$ & \\
\hline 59 & $488-610$ & X07437 \\
\hline 59 & $555-663$ & \\
\hline 59 & $66316-66438$ & AC080013 \\
\hline 59 & 66295-66394 & \\
\hline
\end{tabular}




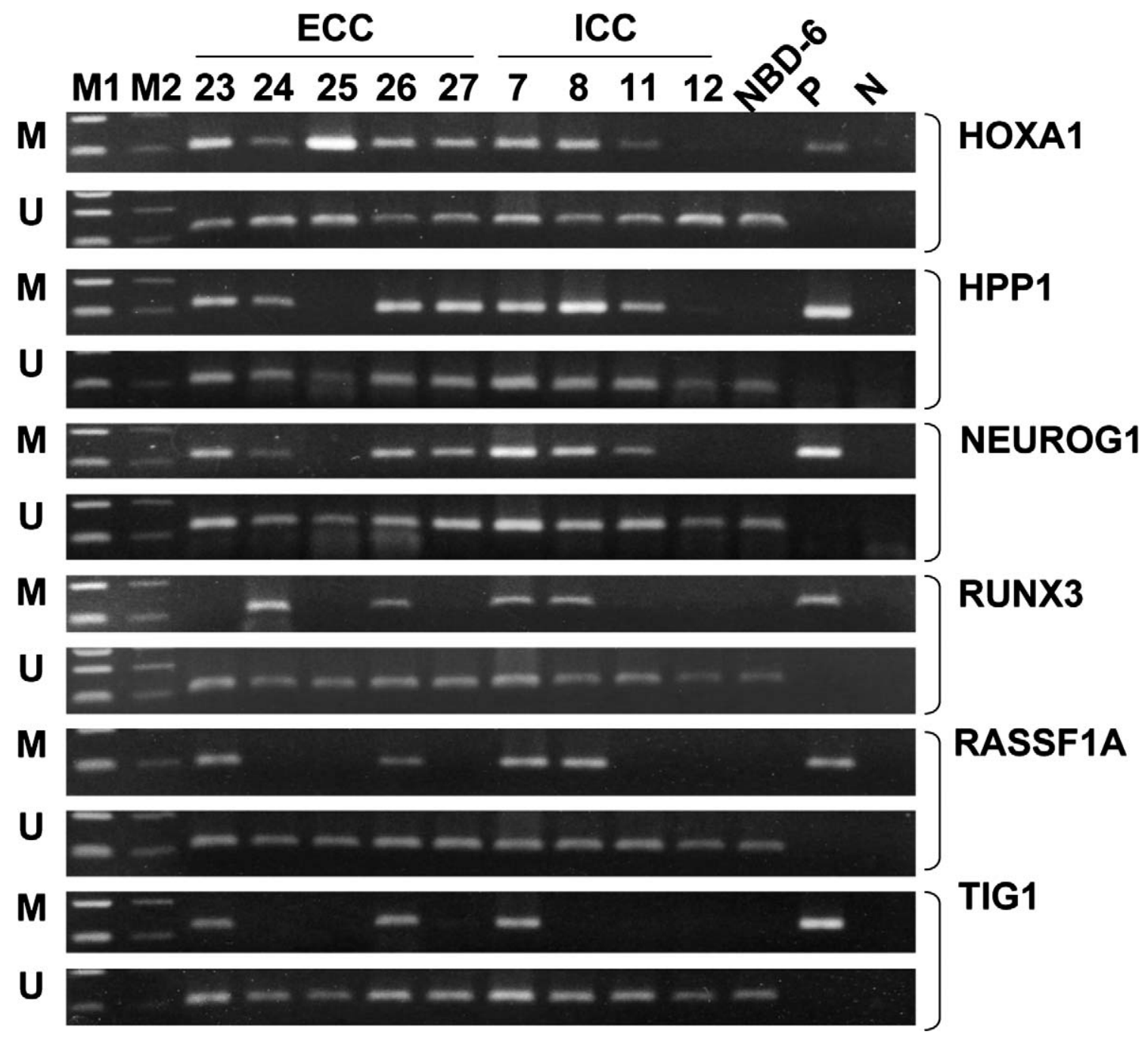

Figure 1. Representative samples of methylation-specific polymerase chain reaction analysis for the methylated $(M)$ and unmethylated $(U)$ forms of 5 most commonly methylated CpG island loci (HOXA1, HPP1, NEUROG1, RUNX3, RASSF1A, and TIG1) in extrahepatic cholangiocarcinomas (ECC), intrahepatic cholangiocarcinomas (ICC), and normal bile duct tissue (NBD). The positive control was a normal lymphocyte DNA treated with Sss 1 methylase before bisulfite modification, and the negative control was distilled water without template DNA. M1 and M2 indicate 100base pair DNA ladder of $14 \mathrm{ng}$ and $3.5 \mathrm{ng}$, respectively; P, positive control; N, negative control.

In ECC, a high frequency of methylation $(>30 \%)$ was detected in HOXA1 $(95.2 \%)$, HPP1 $(69.8 \%)$, NEUROG1 $(61.9 \%)$, CDH1 $(39.7 \%)$, MINT1 $(36.5 \%)$, and RUNX3 (30.2\%), whereas in ICC, HOXA1 (83.3\%), HPP1 (77.1\%), NEUROG1 (41.7\%), MINT1 (39.6\%), RUNX3 (37.5\%), and RASSF1A (31.3\%) displayed high frequencies of methylation. The remaining loci showed methylation frequencies of greater than $30 \%$ in ECC and ICC. The number of loci methylated varied from 0 to 14 in ECC and from 0 to 15 in ICC, with means of 4.9 and 6.0, respectively $(P=.19)$. A comparison of gene methylation frequencies of ECC and ICC showed a statistically significant difference for $C D H 1$, CHFR, GSTP1, IGF2, MGMT, NEUROG1, MINT31, p14, and RBP1. CDH1 and NEUROG1 were more frequently methylated in ECC, whereas CHFR, GSTP1, IGF2, MGMT, MINT31, p14, and RBP1 were more frequently methylated in ICC.

Of $21 \mathrm{CpG}$ island loci methylated in ECC, the loci showing methylation in normal bile ducts included NEUROG1, IGF2, HPP1, CDH1, HOXA1, TIG1, and BCL2 (in decreasing order of hypermethylation frequency). Of these loci,

926 Arch Pathol Lab Med-Vol 131, June 2007
IGF2 did not show a methylation frequency difference between ECC and normal bile duct, but the remaining 6 loci displayed significantly higher hypermethylation frequencies in ECC than in normal bile duct tissues. Concurrent hypermethylation of 3 and $4 \mathrm{CpG}$ island loci was noted in 2 and 1 normal bile duct tissue, respectively. Seven normal bile duct specimens displayed hypermethylation of 1 $\mathrm{CpG}$ island locus. The remainder did not show hypermethylation at the CpG island loci examined. Microscopic examination of these normal control samples confirmed that these biliary epithelia did not have any dysplastic focus. Those 3 normal bile duct samples with concurrent hypermethylation of 3 or $4 \mathrm{CpG}$ island loci were obtained from hepatolithiasis specimens, but the degree of inflammatory cell infiltration in bile ducts did not differ between the hepatolithiasis cases with and without concurrent hypermethylation.

\section{CpG Island Methylation and Clinicopathologic Findings of ECC}

Of the tested $24 \mathrm{CpG}$ island loci, we excluded 12 because they were not methylated in ECC or normal bile 


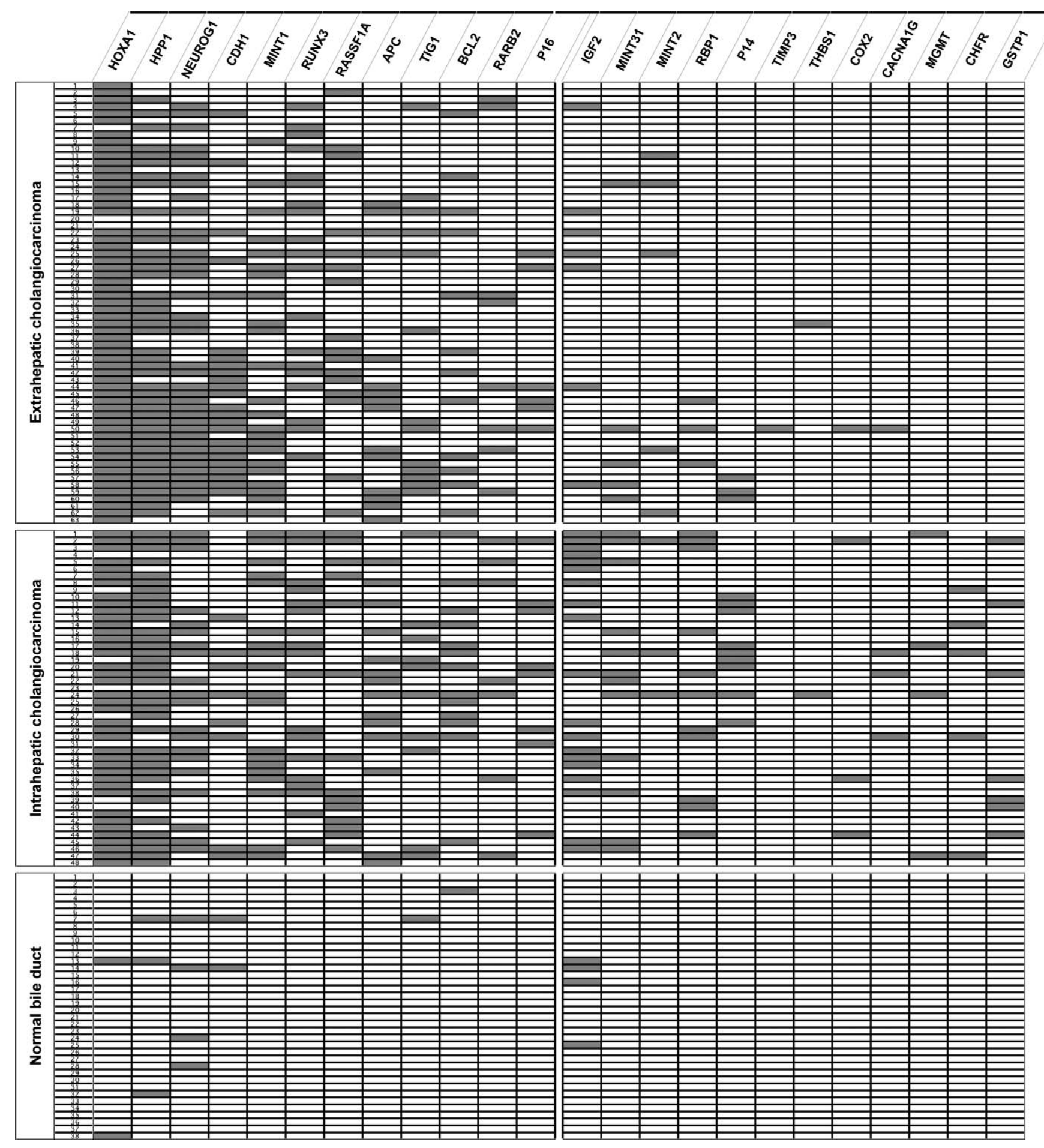

Figure 2. Methylation profile of $24 \mathrm{CpG}$ island loci in extrahepatic cholangiocarcinomas, intrahepatic cholangiocarcinoma, and normal bile duct tissues. A filled box indicates the presence of methylation, and an open box indicates the absence of methylation.

ducts or did not show a statistically significant difference in the methylation frequencies between ECC and normal bile ducts. When the number of methylated $\mathrm{CpG}$ island loci was determined for each gross type, the papillary type showed the highest number of methylated $\mathrm{CpG}$ island loci (5.6/12 CpG island loci) and diffuse type the lowest (3.5 CpG island loci) with scirrhous type (4.9 loci) and nodular type (4.3 loci) intermediate between these two $(P=.13$, analysis of variance test). To determine whether there was

Arch Pathol Lab Med-Vol 131, June 2007 any difference in methylation tendency between proximal and distal ECCs, we compared the methylation frequencies of $\mathrm{CpG}$ island loci between proximal and distal ECCs. $A P C$ and RASSF1A were more frequently methylated in distal ECC (36.7\% vs $12.1 \%, P=.04 ; 40 \%$ vs $12.1 \%, P=$ .02 , respectively). Distal ECC showed a higher number of methylated CpG island loci than did proximal ECC, but this difference was not significant (4.8 vs $4.2, P=.27$, Student $t$ test). When ECCs were divided into low-stage 
Table 3. Methylation Frequencies of 24 CpG Island Loci in Extrahepatic Cholangiocarcinoma (ECC), Intrahepatic Cholangiocarcinoma (ICC), and Normal Bile Ducts (NBD)

\begin{tabular}{|lcclcc|}
\hline \multicolumn{1}{c}{ Loci } & $\begin{array}{c}\text { ECC } \\
(\mathbf{n}=\mathbf{6 3}), \\
\text { No. }(\%)\end{array}$ & $\begin{array}{c}\text { ICC } \\
(\mathbf{n}=\mathbf{4 8}), \\
\text { No. }(\%)\end{array}$ & $\begin{array}{c}\text { NBD } \\
(\mathbf{n}=\mathbf{3 8}), \\
\text { No. }(\%)\end{array}$ & $\begin{array}{c}\boldsymbol{P} \text { Value } \\
\text { ECC vs } \\
\text { ICC }\end{array}$ & $\begin{array}{c}\boldsymbol{P} \text { Value } \\
\text { ECC vs } \\
\text { NBD }\end{array}$ \\
\hline HOXA1 & $60(95.2)$ & $40(83.3)$ & $2(5.2)$ & .05 & $<.001$ \\
HPP1 & $44(69.8)$ & $37(77.1)$ & $3(7.9)$ & .52 & $<.001$ \\
NEUROG1 & $39(61.9)$ & $20(41.7)$ & $4(10.5)$ & .04 & $<.001$ \\
CDH1 & $25(39.7)$ & $8(16.7)$ & $2(5.2)$ & .01 & $<.001$ \\
MINT1 & $23(36.5)$ & $19(39.6)$ & 0 & .84 & $<.001$ \\
RUNX3 & $19(30.2)$ & $18(37.5)$ & 0 & .43 & $<.001$ \\
RASSF1A & $16(25.4)$ & $15(31.3)$ & 0 & .53 & $<.001$ \\
APC & $15(23.8)$ & $14(29.2)$ & 0 & .66 & .001 \\
TIG1 & $14(22.2)$ & $10(20.8)$ & $1(2.6)$ & $>.99$ & .008 \\
BCL2 & $12(19)$ & $13(27.1)$ & $1(2.6)$ & .36 & .03 \\
RARB2 & $8(12.7)$ & $7(14.6)$ & 0 & .79 & .02 \\
p16 & $7(11.1)$ & $8(16.7)$ & 0 & .42 & .04 \\
IGF2 & $7(11.1)$ & $19(39.6)$ & $4(10.5)$ & .001 & $>.99$ \\
MINT31 & $5(7.9)$ & $12(25.0)$ & 0 & .02 & .15 \\
MINT2 & $5(7.9)$ & $3(6.3)$ & 0 & $>.99$ & .15 \\
RBP1 & $4(6.3)$ & $11(22.9)$ & 0 & .02 & .29 \\
p14 & $3(4.8)$ & $9(18.8)$ & 0 & .03 & .29 \\
TIMP3 & $1(1.6)$ & 0 & 0 & $>.99$ & $>.99$ \\
THBS1 & $1(1.6)$ & $1(2.1)$ & 0 & $>.99$ & $>.99$ \\
COX2 & $1(1.6)$ & $3(6.3)$ & 0 & .31 & $>.99$ \\
CACNA1G & $1(1.6)$ & $3(6.3)$ & 0 & .31 & $>.99$ \\
MGMT & 0 & $4(8.3)$ & 0 & .03 & \\
CHFR & 0 & $5(10.4)$ & 0 & .01 & \\
GSTP1 & 0 & $7(14.6)$ & 0 & .002 & \\
\hline
\end{tabular}

(IA, IB, or IIA) and high-stage (IIB, III, or IV) tumors, high-stage ECCs $(n=21)$ exhibited a higher number of methylated CpG island loci than did low-stage ECCs (n $=42$ ); the difference was statistically insignificant (4.9 vs 4.3, $P=.33$, Student $t$ test). BCL2, IGF2, and RUNX3 were more frequently methylated in high-stage ECCs than in low-stage ECCs $(P<.05$, Fisher exact test). Extrahepatic cholangiocarcinomas with nodal metastasis showed a higher number of methylated loci than did ECCs without nodal metastasis (5.5 vs 4.1, $P=.047$, Student $t$ test), and hypermethylation of TIG1 was closely associated with nodal metastasis $(P=.007$, Fisher exact test). However, no association was found between $\mathrm{CpG}$ island hypermethylation and histologic classification or the depth of invasion in terms of hypermethylation of individual CpG island locus or the total number of methylated $\mathrm{CpG}$ island loci. Hypermethylation at any $\mathrm{CpG}$ island locus examined did not affect the clinical outcome of patients with ECC.

\section{COMMENT}

CpG islands are segments of DNA sequences of 0.5 to $2.5 \mathrm{~kb}$ in size that contain clusters of cytosine-guanine dinucleotides and are often located in promoter regions or in the upstream exonal sequences of genes. $\mathrm{CpG}$ islands are usually unmethylated in normal cells, with the exception of those associated with imprinted genes and genes on inactive $\mathrm{X}$ chromosomes. However, $\mathrm{CPG}$ island hypermethylation occurs in relation to cancer development, and almost all tissue types of human cancers display CpG island hypermethylation. Extrahepatic cholangiocarcinoma has been poorly investigated with respect to CpG island hypermethylation, and fewer than 20 genes have been reported to be hypermethylated. In the present study, a panel of $24 \mathrm{CpG}$ island loci was examined with respect to hypermethylation frequency in 63 cases of ECC. Twenty- one of these $24 \mathrm{CpG}$ island loci, except MGMT, CHFR, and GSTP1, exhibited hypermethylation frequencies ranging from $95.2 \%$ to $1.6 \%$, and $96.8 \%$ of 63 ECC cases harbored hypermethylation of at least $1 \mathrm{CpG}$ island locus. Methylation frequencies greater than $10 \%$ were observed in 13 CpG island loci, including HOXA1, HPP1, NEUROG1, CDH1, MINT1, RUNX3, RASSF1A, APC, TIG1, BCL2, RARB2, p16, and IGF2 (in decreasing order of methylation frequency). Twelve CpG island loci, except for IGF2, showed significantly higher methylation frequencies in ECC than in normal bile duct tissues, indicative of a cancer-related change. These findings demonstrate that $\mathrm{CpG}$ island hypermethylation is a frequent finding in ECC, although methylation frequency varied depending on $\mathrm{CpG}$ island locus type.

Although we did not perform a functional study to investigate the inverse relationship between $\mathrm{CpG}$ island hypermethylation and gene expression, the present study represents, to our knowledge, the first report of hypermethylation of HOXA1, HPP1, NEUROG1, MINT1, TIG1, $B C L 2, I G F 2$, and CACNA1G in ECC. These methylation markers, except for $C A C N A 1 G$, showed methylation frequencies greater than $10 \%$ in ECC, and of these, $3 \mathrm{CpG}$ island loci (HOXA1, HPP1, and NEUROG1) showed methylation frequencies of $95.2 \%, 69.8 \%$, and $61.9 \%$, respectively. HOXA1 is one of the A cluster of homeobox genes located on chromosome 7; it encodes a sequence-specific transcription factor that is a component of a developmental regulatory system that provides cells with specific positional identities on the anterior-posterior axis. ${ }^{29}$ Moreover, HOX genes are essential for the regional specification of hollow viscus, such as in the gastrointestinal tract. ${ }^{30}$ HOXA1 has been demonstrated to be expressed in embryonic gastrointestinal tissue and in adult gastrointestinal tissue. $^{31,32}$ However, the methylation status of HOXA1 in various tissue types of human cancers has been unknown, except in lung adenocarcinoma. In a study by Shiraishi et $\mathrm{al}^{33}$ HOXA1 methylation was detected in $62.5 \%(5 / 8)$ of lung adenocarcinomas. When we examined HOXA1 methylation in other tissue types of human cancers, its methylation frequencies were found to be $51.4 \%(18 / 35), 11.8 \%$ $(17 / 144)$, and $0(0 / 179)$ in gastric cancer, colorectal cancer, and prostate cancer, respectively (data not shown). HPP1 encodes a transmembrane protein with epithelial growth factor-like and 2 follistatin-like domains 2, and may function as both a growth factor and a receptor. ${ }^{34,35}$ HPP1 was initially identified because it is frequently hypermethylated in hyperplastic polyps of the colon. ${ }^{36}$ It has been demonstrated to be hypermethylated in colorectal cancer, gastric cancer, ${ }^{37}$ esophageal adenocarcinoma, ${ }^{38}$ lung cancer ${ }^{39}$ gallbladder cancer, ${ }^{40}$ and prostate cancer. ${ }^{41}$ On the other hand, NEUROG1 encodes a basic helix-loop-helix family transcription factor, which induces neurogenesis and inhibits the differentiation of neural stem cells into astrocytes. ${ }^{42,43}$ Little is known about the prevalence of NEUROG1 hypermethylation except in colorectal cancer. ${ }^{44}$

The poor prognosis of ECC is largely attributable to an advanced disease stage at presentation. Early detection and resection would undoubtedly improve patient survival. However, because endobiliary brushing cytology and tissue biopsy allow cancer detection in less than half of ECC cases ${ }^{45}$ molecular biology techniques have been applied to improve detection sensitivity. KRAS mutation is one of the genetic alterations commonly studied with respect to its sensitivity and specificity for the detection of 
tumor cells in bile juice. However, the frequency of $K R A S$ mutation is not high; reported mutation rates vary from $8.3 \%$ to $75 \%{ }^{46,47}$ In the present study, HOXA1 displayed a methylation frequency of $95.2 \%$ in ECC and provided positive and negative prediction values of $97 \%$ and $92 \%$, respectively. These findings indicate that HOXA1 methylation is a sensitive and specific marker for ECC. Thus, HOXA1 methylation has a promising potential value as a molecular marker for detecting ECC cells in bile juice. A large-scale case-control study is required to assess the merits of HOXA1 methylation as a biomarker for the differentiation of benign and malignant strictures in extrahepatic bile ducts.

Previously, Yang et al ${ }^{18}$ investigated the methylation profiles of 12 CpG island loci in 36 ECC cases and 36 ICC cases and found that they had overlapping but distinct methylation profiles, which is consistent with our findings. When the $\mathrm{CpG}$ island locus methylation frequencies in ECC in the study by Yang et al and in the present study were compared, the methylation frequencies did not differ for CDH1, GSTP1, and RARB2 but greatly differed for RASSF1A, p16, APC, p14, and MGMT. The latter 4 CpG island loci showed methylation frequencies of $40 \%$ or more in the study by Yang et al but of less than 30\% in the present study. This discrepancy might be attributed to differences in the methylation assay methods used. Yang et al adopted a nested 2-step MSP approach, which gave them a higher sensitivity for detecting allelic hypermethylation in targeted sequences than that offered by the traditional single-step MSP. Other factors include differences in the CpG sites examined and ethnic differences between the study populations.

Forty-eight cases of ICC, in which the methylation statuses had been studied before, ${ }^{26}$ were included again in this study. However, our previous study and the present study significantly differed in terms of the frequency of methylation for MINT31: methylation frequency for MINT31 was $1.3 \%$ previously and $25 \%$ in the present study. The inconsistent data were attributable to the fact that oligonucleotide primer sets for MINT2 and MINT31 ${ }^{28}$ in the present study were different from those ${ }^{26}$ in a previous study. Because we analyzed DNA samples obtained from formalin-fixed, paraffin-embedded tissue, amplicon size was important and should be kept as short as possible. We chose the new sets of primers because the new sets amplify shorter genomic fragments; we confirmed that in a preliminary study, the new sets gave more frequent and stronger signals in comparison with the old sets.

In conclusion, in the present study, we examined 24 CpG island loci in 63 ECCs and 48 ICCs for methylation status by MSP, and we found that $\mathrm{CpG}$ island hypermethylation is a frequent finding in ECC and ICC. Moreover, ICC and ECC tissues exhibited overlapping but distinct methylation profiles. In ECC, a close relationship existed between $\mathrm{CPG}$ island hypermethylation and nodal metastasis. In addition, HOXA1 was frequently methylated in ECC, which raises the possibility that HOXA1 methylation might be used as a molecular marker for the differentiation of malignant and benign biliary strictures.

This study was supported by the Cancer Research Institute, Seoul National University College of Medicine (No. CRI-05-02), and by the second stage Brain Korea 21 project.

\section{References}

1. Jemal A, Murray T, Samuels A, Ghafoor A, Ward E, Thun MJ. Cancer statistics, 2003. CA Cancer J Clin. 2003;53:5-26.
2. Pitt HA, Dooley WC, Yeo CJ, Cameron JL. Malignancies of the biliary tree. Curr Probl Surg. 1995;32:1-90.

3. Henson DE, Albores-Saavedra J, Corle D. Carcinoma of the extrahepatic bile ducts: histologic types, stage of disease, grade, and survival rates. Cancer. 1992;70:1498-1501.

4. Scarpa A, Capelli P, Zamboni G, et al. Neoplasia of the ampulla of Vater: Ki-ras and p53 mutations. Am J Pathol. 1993;142:1163-1172.

5. Suto T, Habano W, Sugai T, et al. Aberrations of the K-ras, p53, and APC genes in extrahepatic bile duct cancer. J Surg Oncol. 2000;73:158-163.

6. Nakazawa K, Dobashi Y, Suzuki S, Fujii H, Takeda Y, Ooi A. Amplification and overexpression of c-erbB-2, epidermal growth factor receptor, and c-met in biliary tract cancers. J Pathol. 2005;206:356-365.

7. Suto T, Sugai T, Habano W, et al. Allelotype analysis of the PTEN, Smad4 and DCC genes in biliary tract cancer. Anticancer Res. 2002;22:1529-1536.

8. Costello JF, Fruhwald MC, Smiraglia DJ, et al. Aberrant CpG-island methylation has non-random and tumour-type-specific patterns. Nat Genet. 2000;24: 132-138.

9. Esteller M, Corn PG, Baylin SB, Herman JG. A gene hypermethylation profile of human cancer. Cancer Res. 2001;61:3225-3229.

10. Virmani AK, Tsou JA, Siegmund KD, et al. Hierarchical clustering of lung cancer cell lines using DNA methylation markers. Cancer Epidemiol Biomarkers Prev. 2002;11:291-297.

11. Caca K, Feisthammel J, Klee K, et al. Inactivation of the INK4a/ARF locus and p53 in sporadic extrahepatic bile duct cancers and bile tract cancer cell lines. Int J Cancer. 2002;97:481-488.

12. Chen YJ, Tang QB, Zou SQ. Inactivation of RASSF1A, the tumor suppressor gene at 3p21.3 in extrahepatic cholangiocarcinoma. World J Gastroenterol.2005; 11:1333-1338.

13. Kim SG, Chan AO, Wu TT, Issa JP, Hamilton SR, Rashid A. Epigenetic and genetic alterations in duodenal carcinomas are distinct from biliary and ampullary carcinomas. Gastroenterology. 2003;124:1300-1310.

14. Koga Y, Kitajima Y, Miyoshi A, et al. Tumor progression through epigenetic gene silencing of $\mathrm{O}(6)$-methylguanine-DNA methyltransferase in human biliary tract cancers. Ann Surg Oncol. 2005;12:354-363.

15. Ku JL, Yoon KA, Kim IJ, et al. Establishment and characterization of six human biliary tract cancer cell lines. Br J Cancer. 2002;87:187-193.

16. Sorio C, Moore PS, Ennas MG, et al. A novel cell line and xenograft model of ampulla of Vater adenocarcinoma. Virchows Arch. 2004;444:269-277.

17. Ueki T, Hsing AW, Gao YT, et al. Alterations of $\mathrm{p} 16$ and prognosis in biliary tract cancers from a population-based study in China. Clin Cancer Res. 2004;10: $1717-1725$

18. Yang B, House MG, Guo M, Herman JG, Clark DP. Promoter methylation profiles of tumor suppressor genes in intrahepatic and extrahepatic cholangiocarcinoma. Mod Pathol. 2005; 18:412-420.

19. Eads CA, Lord RV, Wickramasinghe K, et al. Epigenetic patterns in the progression of esophageal adenocarcinoma. Cancer Res. 2001;61:3410-3418.

20. Soria JC, Rodriguez M, Liu DD, Lee Jl, Hong WK, Mao L. Aberrant promoter methylation of multiple genes in bronchial brush samples from former cigarette smokers. Cancer Res. 2002;62:351-355.

21. Howell DA, Parsons WG, Jones MA, Bosco JJ, Hanson BL. Complete tissue sampling of biliary strictures at ERCP using a new device. Gastrointest Endosc. 1996;43:498-502.

22. Kipp BR, Stadheim LM, Halling SA, et al. A comparison of routine cytology and fluorescence in situ hybridization for the detection of malignant bile duct strictures. Am J Gastroenterol. 2004;99:1675-1681.

23. Yamaguchi K, Nakano K, Nagai E, et al. Ki-ras mutations in codon 12 and p53 mutations (biomarkers) and cytology in bile in patients with hepatobiliarypancreatic carcinoma. Hepatogastroenterology. 2005;52:713-718.

24. Greene GL, Page DL, Fleming ID, et al, eds. Digestive system: extrahepatic bile ducts. In: AJCC Cancer Staging Manual. New York, NY: Springer-Verlag; 2002:145-150.

25. Hamilton RS, Aaltonen LA, eds. Pathology and Genetics of Tumours of the Digestive System. Lyon, France: IARC Press; 2000:314. World Health Organization Classification of Tumours.

26. Lee $\mathrm{S}$, Kim $\mathrm{WH}$, Jung HY, Yang MH, Kang GH. Aberrant $\mathrm{CpG}$ island methylation of multiple genes in intrahepatic cholangiocarcinoma. Am J Pathol. 2002; 161:1015-1022.

27. Kang GH, Lee HJ, Kim JH, et al. Aberrant CpG island hypermethylation along multistep hepatocarcinogenesis. Am J Pathol. 2003;163:1371-1378.

28. Park SJ, Rashid A, Lee JH, Kim SG, Hamilton SR, Wu TT. Frequent CpG island methylation in serrated adenomas of the colorectum. Am J Pathol. 2003; 162:815-822.

29. McGinnis W, Krumlauf R. Homeobox genes and axial patterning. Cell. 1992;68:283-302.

30. Roberts DJ, Smith DM, Goff DJ, Tabin CJ. Epithelial-mesenchymal signaling during the regionalization of the chick gut. Development. 1998;125:2791-2801.

31. Kawazoe Y, Sekimoto T, Araki M, Takagi K, Araki K, Yamamura K. Regionspecific gastrointestinal Hox code during murine embryonal gut development. Dev Growth Differ. 2002;44:77-84.

32. Yahagi N, Kosaki R, Ito T, et al. Position-specific expression of Hox genes along the gastrointestinal tract. Congenit Anom (Kyoto). 2004;44:18-26.

33. Shiraishi M, Sekiguchi A, Oates AJ, Terry MJ, Miyamoto Y. HOX gene clusters are hotspots of de novo methylation in $\mathrm{CpG}$ islands of human lung adenocarcinomas. Oncogene. 2002;21:3659-3662.

34. Uchida T, Wada K, Akamatsu T, et al. A novel epidermal growth factor- 
like molecule containing two follistatin modules stimulates tyrosine phosphorylation of erbB-4 in MKN28 gastric cancer cells. Biochem Biophys Res Commun. 1999;266:593-602.

35. Horie $M$, Mitsumoto $Y$, Kyushiki $H$, et al. Identification and characterization of TMEFF2, a novel survival factor for hippocampal and mesencephalic neurons. Genomics. 2000;67:146-152.

36. Young J, Biden KG, Simms LA, et al. HPP1: a transmembrane proteinencoding gene commonly methylated in colorectal polyps and cancers. Gut. 2006. Epub ahead of print.

37. Shibata DM, Sato F, Mori Y, et al. Hypermethylation of HPP1 is associated with hMLH1 hypermethylation in gastric adenocarcinomas. Cancer Res. 2002; 62:5637-5640.

38. Geddert $\mathrm{H}$, Kiel $\mathrm{S}$, Iskender $\mathrm{E}$, et al. Correlation of hMLH1 and HPP1 hypermethylation in gastric, but not in esophageal and cardiac adenocarcinoma. Int J Cancer. 2004;110:208-211.

39. Hanabata T, Tsukuda K, Toyooka S, et al. DNA methylation of multiple genes and clinicopathological relationship of non-small cell lung cancers. Oncol Rep. 2004;12:177-180.

40. Takahashi T, Shivapurkar N, Riquelme E, et al. Aberrant promoter hypermethylation of multiple genes in gallbladder carcinoma and chronic cholecystitis. Clin Cancer Res. 2004;10:6126-6133.
41. Suzuki M, Shigematsu H, Shivapurkar N, et al. Methylation of apoptosis related genes in the pathogenesis and prognosis of prostate cancer. Cancer Lett. 2006. Epub ahead of print.

2. McCormick MB, Tamimi RM, Snider L, Asakura A, Bergstrom D, Tapscott SJ. NeuroD2 and neuroD3: distinct expression patterns and transcriptional activation potentials within the neuroD gene family. Mol Cell Biol. 1996;16:57925800

3. Sun $Y$, Nadal-Vicens $M$, Misono $S$, et al. Neurogenin promotes neurogenesis and inhibits glial differentiation by independent mechanisms. Cell. 2001;104: 365-376.

44. Ogino S, Cantor M, Kawasaki T, et al. CpG island methylator phenotype (CIMP) of colorectal cancer is best characterized by quantitative DNA methylation analysis and prospective cohort studies. Gut. 2006. Epub ahead of print.

45. Ponchon T, Gagnon P, Berger F, et al. Related value of endobiliary brush cytology and biopsies for the diagnosis of malignant bile duct stenosis: results of a prospective study. Gastrointest Endosc. 1995;42:565-572.

46. Tada M, Yokosuka O, Omata M, Ohto M, Isono K. Analysis of ras gene mutations in biliary and pancreatic tumors by polymerase chain reaction and direct sequencing. Cancer. 1990;66:930-935.

47. Rijken AM, van Gulik TM, Polak MM, Sturm PD, Gouma DJ, Offerhaus G). Diagnostic and prognostic value of incidence of K-ras codon 12 mutations in resected distal bile duct carcinoma. J Surg Oncol. 1998;68:187-192. 
Reproduced with permission of the copyright owner. Further reproduction prohibited without permission. 